\title{
Molecular mechanisms of pancreatic stone formation in chronic pancreatitis
} \author{
Minoru S. H. Ko ${ }^{1}$ and Hiroshi Ishiguro ${ }^{4}$ \\ ' Sakaguchi Laboratory, Department of Systems Medicine, Keio University School of Medicine, Tokyo, Japan \\ 2 Department of Gastroenterology, National Center for Geriatrics and Gerontology, Obu, Japan \\ ${ }^{3}$ Yoshikawa Clinic, Shizuoka, Japan \\ ${ }^{4}$ Department of Human Nutrition, Nagoya University Graduate School of Medicine, Nagoya, Japan
}

Shigeru B. H. Ko ${ }^{1 *}$, Sakiko Azuma ${ }^{2}$, Toshiyuki Yoshikawa ${ }^{3}$, Akiko Yamamoto $^{4}$, Kazuhiro Kyokane ${ }^{2}$,

\section{Edited by:}

Atsushi Masamune, Tohoku University Graduate School of Medicine, Japan

Reviewed by:

Masaki Ohmuraya, Kumamoto University, Japan

Kiyoshi Kume, Tohoku University

Graduate School of Medicine, Japan

*Correspondence:

Shigeru B. H. Ko, Sakaguchi

Laboratory, Department of Systems

Medicine, Keio University School of

Medicine, Tokyo 160-8582, Japan.

e-mail:kos@a6.keio.jp
Chronic pancreatitis (CP) is a progressive inflammatory disease in which the pancreatic secretory parenchyma is destroyed and replaced by fibrosis. The presence of intraductal pancreatic stone(s) is important for the diagnosis of $\mathrm{CP}$; however, the precise molecular mechanisms of pancreatic stone formation in $\mathrm{CP}$ were left largely unknown. Cystic fibrosis transmembrane conductance regulator (CFTR) is a chloride channel expressed in the apical plasma membrane of pancreatic duct cells and plays a central role in $\mathrm{HCO}_{3}^{-}$secretion. In previous studies, we have found that CFTR is largely mislocalized to the cytoplasm of pancreatic duct cells in all forms of CP and corticosteroids normalizes the localization of CFTR to the proper apical membrane at least in autoimmune pancreatitis. From these observations, we could conclude that the mislocalization of CFTR is a cause of protein plug formation in $\mathrm{CP}$, subsequently resulting in pancreatic stone formation. Considering our observation that the mislocalization of CFTR also occurs in alcoholic or idiopathic $\mathrm{CP}$, it is very likely that these pathological conditions can also be treated by corticosteroids, thereby preventing pancreatic stone formation in these patients. Further studies are definitely required to clarify these fundamental issues.

Keywords: chronic pancreatitis, pancreatic stone formation, bicarbonate secretion, CFTR, cytoplasmic mislocalization

\section{INTRODUCTION}

Chronic pancreatitis $(\mathrm{CP})$ is a progressive inflammatory disease of the pancreas, and is characterized by pancreatic exocrine and endocrine dysfunction resulting from tissue damage caused by inflammation. The pancreatic exocrine gland is composed of two types of cells, duct cells and acinar cells. Duct cells secrete fluid and $\mathrm{HCO}_{3}^{-}$to neutralize gastric acid from the stomach. Acinar cells secrete digestive enzymes essential for the digestion of food. Regardless of the cause of pancreatitis, $\mathrm{HCO}_{3}^{-}$and digestive enzyme secretion are more or less compromised in all forms of chronic pancreatitis.

For a diagnosis of CP (Homma et al., 1997), it is essential to show the inflammation and destruction of the gland; however, it is often difficult to obtain pancreatic tissues due to the anatomy of the gland, except in cases of a pancreatic resection for malignant tumors. Another way to diagnose $\mathrm{CP}$ is to show exocrine pancreatic dysfunction. Pancreatic ductal dysfunction, especially a low $\mathrm{HCO}_{3}^{-}$concentration in the pancreatic juice, is the most important finding for the diagnosis of $\mathrm{CP}$ with mild or moderate exocrine dysfunction, since an impairment in ductal $\mathrm{HCO}_{3}^{-}$ secretion is one of the earliest defects in CP (Freedman, 1998). For evaluating exocrine function, the secretin test was the only reliable test which can directly measure pancreatic ductal function (Ko et al., 2010) and acinar cell function separately; however, it became impossible to diagnose mild or moderate CP Functionally because the secretin test is no longer available in Japan due to the lack of supply of the clinical grade secretin. For that reason, the presence of pancreatic ductal stones became the most reliable diagnostic criterion for chronic pancreatitis.

Pancreatic stones are thought to be formed at first as protein plugs in pancreatic ducts in CP (Freedman, 1998). However, detailed molecular mechanisms of how pancreatic stones are formed in pancreatic ducts of $\mathrm{CP}$ still remain elusive. In previous studies, while elucidating the molecular mechanisms of aberrant $\mathrm{HCO}_{3}^{-}$transport in pancreatic ducts in chronic pancreatitis, we have found that the Cystic fibrosis transmembrane conductance regulator (CFTR) chloride channel, which plays a central role in $\mathrm{HCO}_{3}^{-}$transport in pancreatic ducts, is largely mislocalized in the cytoplasm of pancreatic duct cells in autoimmune pancreatitis (Ko et al., 2010). As the cytoplasmic mislocalization of CFTR has been observed in all other forms of CP (i.e., alcoholic, idiopathic, or obstructive), we concluded that the mislocalization of CFTR is a cause of pancreatic ductal dysfunction and subsequent pancreatic stone formation. This notion was further supported by the observation that corticosteroids, a potent anti-inflammatory drug, normalize the CFTR localization from the cytoplasm of pancreatic duct cells to the proper apical plasma membrane, and subsequently restored aberrant pancreatic $\mathrm{HCO}_{3}^{-}$secretion.

In this article, we propose that the cytoplasmic mislocalization of the CFTR in pancreatic duct cells is a cause of pancreatic 


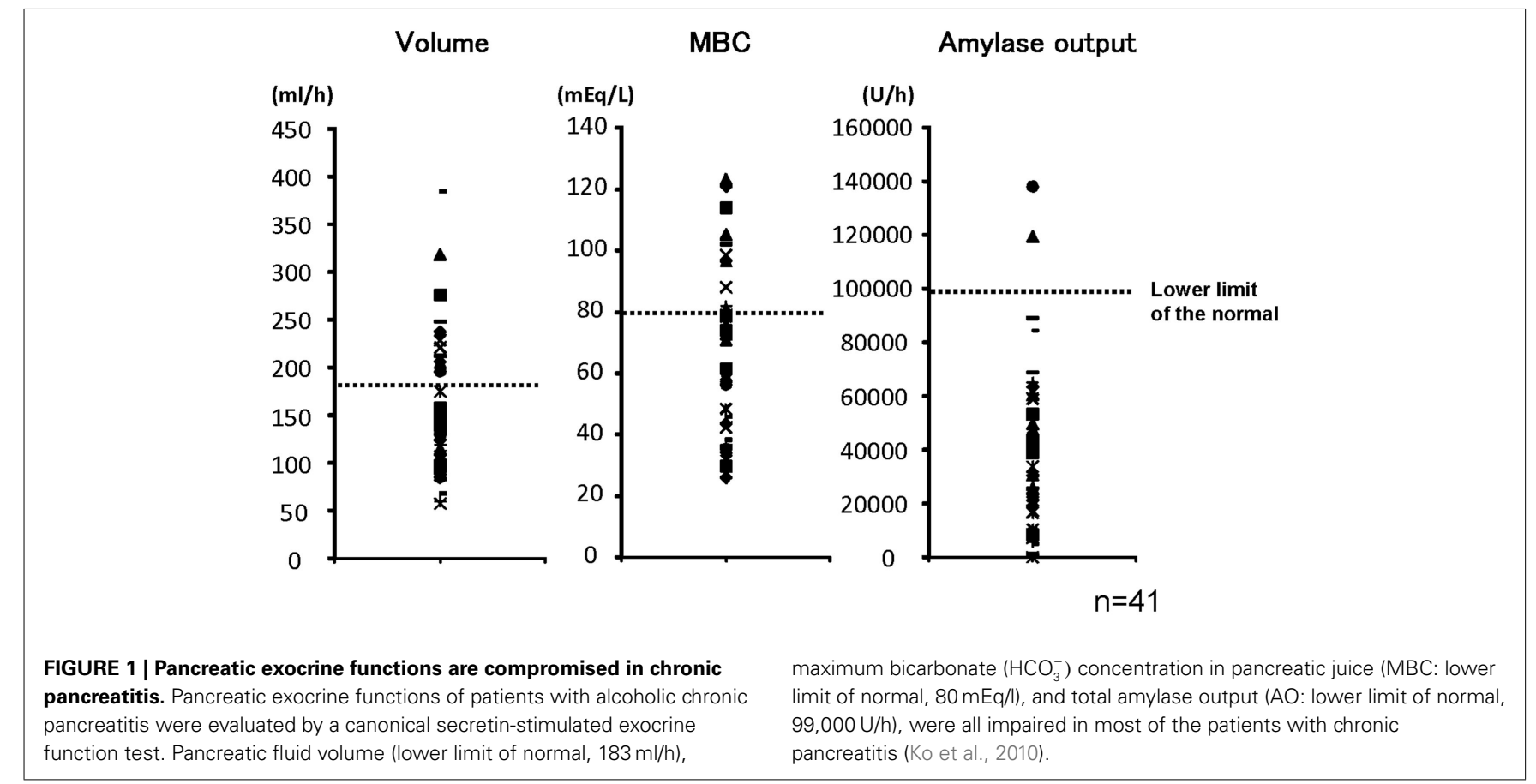

stone formation in chronic pancreatitis. Currently, steroid treatment for autoimmune pancreatitis is the only established therapy to restore impaired pancreatic ductal function. It might be possible that pancreatic ductal dysfunction seen in alcoholic or idiopathic pancreatitis can also be cured by the administration of corticosteroid treatment. Further studies are definitely required to clarify these important issues in the treatment of chronic pancreatitis.

\section{$\mathrm{HCO}_{3}^{-}$SECRETION FROM PANCREATIC DUCT CELLS IS COMPROMISED IN CHRONIC PANCREATITIS}

The normal pancreas secretes the most alkaline fluid among exocrine organs in humans (maximum $\mathrm{HCO}_{3}^{-}$concentration in pancreatic juice around $140 \mathrm{mM}, \mathrm{pH} \sim 8.5$ ) to neutralize acid from the stomach (Steward et al., 2005). In pancreatic ducts in chronic pancreatitis, it is well known that the alkalinization of pancreatic juice is impaired, and results in a low $\mathrm{pH}$ in secreted fluid. However, the precise molecular mechanisms of pancreatic ductal dysfunction remained elusive.

Figure 1 shows pancreatic exocrine functions in patients with alcoholic chronic pancreatitis. Pancreatic fluid volume (V: lower limit of normal, $183 \mathrm{ml} / \mathrm{h}$ ), maximum bicarbonate concentration (MBC) in pancreatic juice (lower limit of normal, $80 \mathrm{mEq} / \mathrm{l}$ ), and total amylase output (AO: lower limit of normal, 99,000 U/h), were all impaired in alcoholic chronic pancreatitis. In a severe form of chronic pancreatitis, all three factors are impaired. In some cases with mild or moderate forms of chronic pancreatitis, fluid volume, and AO may stay within normal range; however, $\mathrm{HCO}_{3}^{-}$secretion is frequently impaired even in a mild form of the disease. Therefore direct measurement of maximum $\mathrm{HCO}_{3}^{-}$ concentration in pancreatic juice was very valuable to diagnose mild or moderate forms of CP when the secretin test was available.

\section{MOLECULAR MECHANISM OF PANCREATIC $\mathrm{HCO}_{3}^{-}$ SECRETION FROM THE NORMAL DUCT CELLS}

The pancreatic duct epithelium is capable of secreting $\mathrm{HCO}_{3}^{-}$at a concentration of around $140 \mathrm{mM}$ (Steward et al., 2005). Molecular mechanisms of how the pancreatic duct epithelium secretes such a high concentration of $\mathrm{HCO}_{3}^{-}$have long been examined (Figure 2). A primary fluid rich in digestive enzymes secreted from pancreatic acinar cells contains around $24 \mathrm{mM} \mathrm{HCO}_{3}^{-}$. The digestive hormone secretin was secreted from the endocrine cells in the duodenum when these cells were stimulated with gastric acid from the stomach. Secretin binds to its receptor on the basolateral membrane of pancreatic duct cells. An increase of intracellular cyclic AMP levels stimulates the CFTR chloride channel which is located at the apical plasma membrane of small pancreatic duct cells. $\mathrm{Cl}^{-}$ions pass through the CFTR chloride channel into the luminal space of pancreatic ducts. $\mathrm{HCO}_{3}^{-}$was secreted from pancreatic duct cells in exchange for $\mathrm{Cl}^{-}$absorption by an electrogenic anion exchanger, SLC26 transporters, expressed also at the apical plasma membrane of pancreatic ducts (Ko et al., 2002, 2004; Song et al., 2012). When $\mathrm{Cl}^{-}$concentration in the juice becomes quite low, $\mathrm{HCO}_{3}^{-}$was secreted to the luminal space of pancreatic ducts through CFTR chloride channels (Ishiguro et al., 2009).

\section{CFTR CHLORIDE CHANNEL IS MISLOCALIZED TO THE CYTOPLASM OF PANCREATIC DUCTS IN CHRONIC PANCREATITIS}

It has been well known that the $\mathrm{HCO}_{3}^{-}$concentration in pancreatic juice is reduced in CP (Braganza et al., 2011). In previous studies, however, it was unclear why $\mathrm{HCO}_{3}^{-}$concentration in pancreatic juice in $\mathrm{CP}$ is low. It has been shown that the CFTR plays a most pivotal role in $\mathrm{HCO}_{3}^{-}$secretion in pancreatic duct cells. Thus, it is reasonable to consider whether or not the expression of CFTR is 


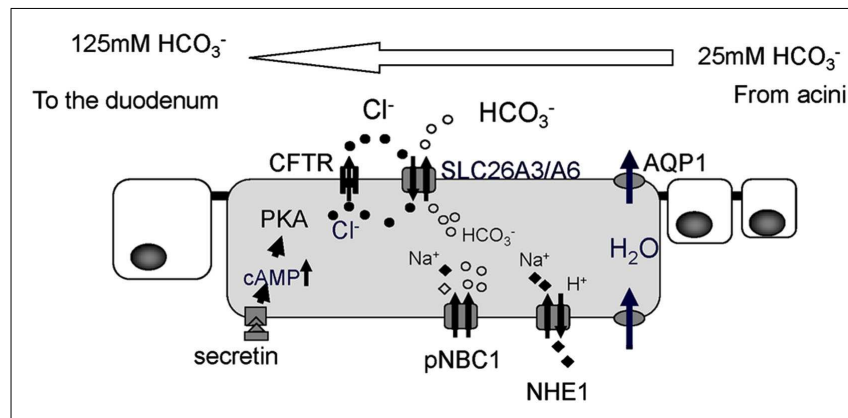

FIGURE 2 | Cellular mechanism proposed for ion and fluid secretion by the pancreatic duct epithelium. The primary fluid secreted by acinar cells contains a large amount of digestive enzymes and $24 \mathrm{mM} \mathrm{HCO}_{3}^{-}$. Intracellular $\mathrm{HCO}_{3}^{-}$leaves the cell in exchange for $\mathrm{Cl}^{-}$, mediated by SLC26 anion exchangers. $\mathrm{Cl}^{-}$is supplied to the lumen by a secretin-stimulated $\mathrm{Cl}^{-}$ channel, the Cystic fibrosis transmembrane conductance regulator (CFTR). For fluid movement across the epithelium, aquaporin (AOP) water channels are present at both the apical and basolateral membranes of smaller ducts in the rat (Furuya et al., 2002) and human pancreas (Burghardt et al., 2003). PKA, protein kinase $A ;$ pNBC1, pancreatic sodium bicarbonate cotransporter1; NHE1, sodium proton exchanger1.

compromised in pancreatic duct cells in patients with chronic pancreatitis. To this end, we have examined the immunolocalization of the CFTR in these patients. In the normal pancreas the CFTR is expressed exclusively at the plasma membrane of small pancreatic ducts (Figure 3). However, in chronic pancreatitis, trafficking of the CFTR is largely compromised and the protein is largely retained at the cytoplasm of pancreatic ducts (Figure 3). CFTR plays a central role in $\mathrm{HCO}_{3}^{-}$secretion from pancreatic duct cells; therefore, the reduced CFTR expression at the apical membrane of pancreatic ducts should result in the low $\mathrm{HCO}_{3}^{-}$concentration seen in CP (Ko et al., 2011).

\section{STEROID THERAPY NORMALIZES CYTOPLASMIC MISLOCALIZATION OF CFTR TO THE APICAL PLASMA MEMBRANE OF PANCREATIC DUCT CELLS IN AUTOIMMUNE PANCREATITIS AND RECOVERS ABERRANT $\mathrm{HCO}_{3}^{-}$ SECRETION IN CHRONIC PANCREATITIS}

Autoimmune pancreatitis is one form of chronic pancreatitis. Autoimmunity is suspected to be its pathogenesis (Yoshida et al., 1995). Severe exocrine insufficiency has been reported in most of the cases (Ito et al., 2007; Frulloni et al., 2010; Ko et al., 2010). Steroid therapy improves the swelling of the gland and narrowing of the main pancreatic ducts, and reduces serum gamma globulin and immunoglobulin G subtype 4 (IgG4) levels.

To further elucidate the role of CFTR mislocalization in the aberrant ductal $\mathrm{HCO}_{3}^{-}$secretion in chronic pancreatitis, we have examined histology (Mizuno et al., 2009) and exocrine functions of patients with autoimmune pancreatitis at the diagnosis and 3 months after maintenance steroid treatment (Ko et al., 2010). In autoimmune pancreatitis, $\mathrm{HCO}_{3}^{-}$concentration in pancreatic juice is remarkably reduced prior to treatment as well as in other forms of CP (Figure 4), whereas 3 months of steroid therapy restored the mislocalization of the CFTR to the proper apical plasma membrane and significantly improved the $\mathrm{HCO}_{3}^{-}$

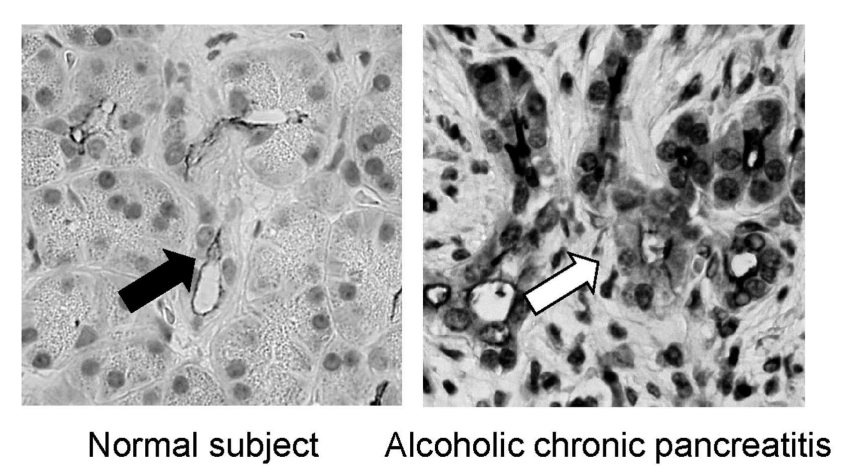

FIGURE 3 | Immnolocalization of CFTR in the pancreas of normal and chronic pancreatitis. In the normal subjects, the CFTR chloride channel is exclusively localized in the apical plasma membrane of small pancreatic ducts (left panel). In contrast, the CFTR chloride channel is largely retained at the cytoplasm of pancreatic ducts and is not transported to the proper apical plasma membrane domain in alcoholic chronic pancreatitis (right panel; modified from Freedman, 1998).

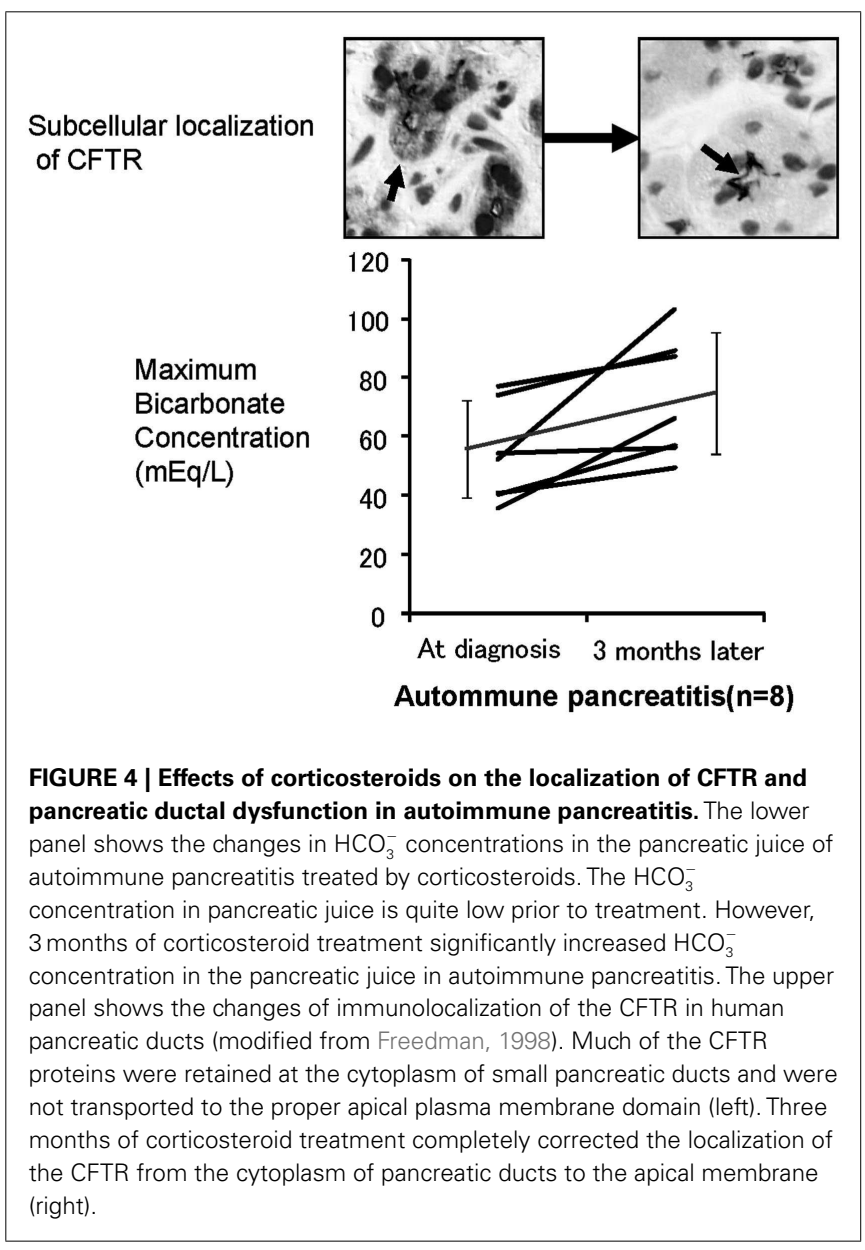

concentration in pancreatic juice (Figure 4). These data indicate that the mislocalization of the CFTR is a direct cause of a low $\mathrm{HCO}_{3}^{-}$concentration in pancreatic juice and that steroid 
treatment restores both the localization of CFTR and $\mathrm{HCO}_{3}^{-}$ concentration in pancreatic juice in autoimmune pancreatitis.

\section{PANCREATIC DUCTAL DYSFUNCTION IS NOT RECOVERED} SPONTANEOUSLY IN CHRONIC ALCOHOLIC PANCREATITIS

We have shown that the mislocalization of the CFTR in the cytoplasm of pancreatic ducts is a possible cause of the low

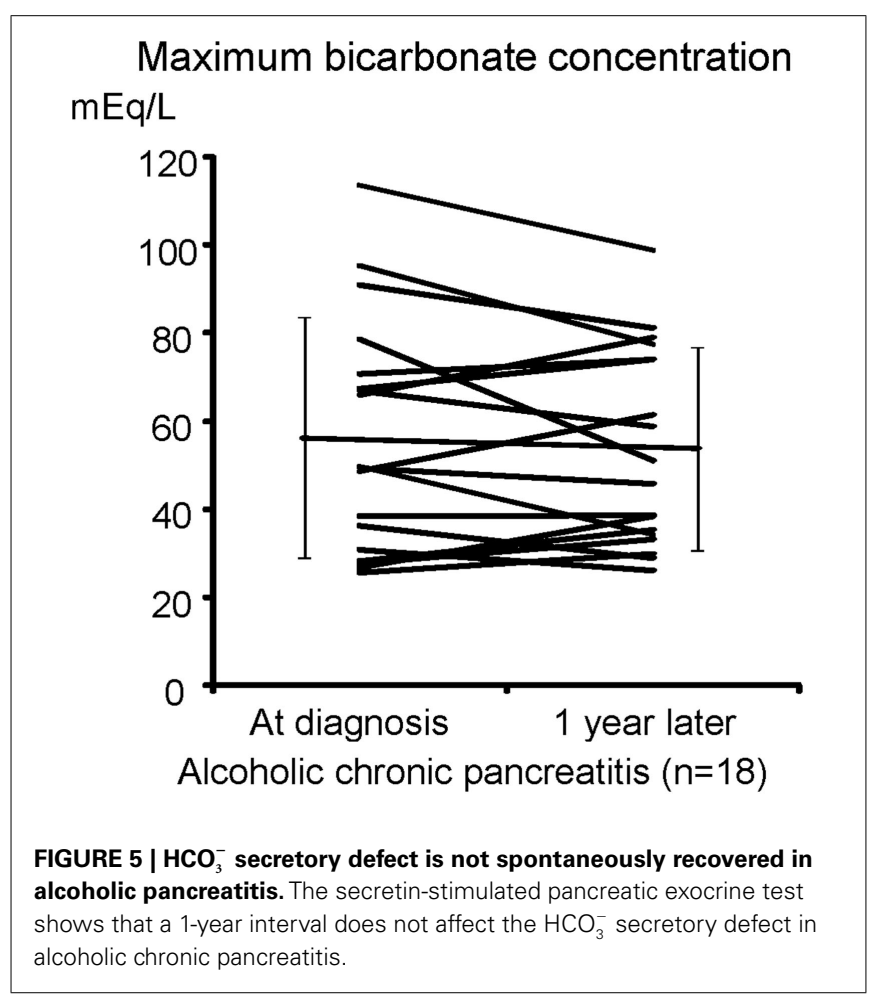

$\mathrm{HCO}_{3}^{-}$concentration found in pancreatic juice in chronic pancreatitis. If this proposed molecular mechanism is correct, one would speculate that the retargeting of the CFTR at the apical plasma membrane by the specific anti-inflammatory treatment will restore pancreatic $\mathrm{HCO}_{3}^{-}$secretion in chronic pancreatitis, and prevent pancreatic stone formation in chronic pancreatitis. Thus, we next examined $\mathrm{HCO}_{3}^{-}$concentrations in the juice of 18 cases of chronic alcoholic pancreatitis to see if pancreatic ductal dysfunction spontaneously recovers (Figure 5). As shown in Figure 5, a 1-year observation period did not affect the $\mathrm{HCO}_{3}^{-}$ concentrations of pancreatic juice in chronic alcoholic pancreatitis without an active anti-inflammatory regimen, indicating that pancreatic ductal dysfunction does not improve spontaneously in chronic pancreatitis.

\section{PROPOSED MOLECULAR MECHANISM OF PANCREATIC STONE FORMATION IN CHRONIC PANCREATITIS}

Figure 6 shows the possible steps/mechanisms for pancreatic stone formation in chronic pancreatitis.

Step 1: Chronic inflammation occurred in the pancreas by drinking, gallstone, or autoimmune mechanisms.

Step 2: Trafficking of membrane proteins such as the CFTR is compromised and the proteins are mislocalized to the cytoplasm of pancreatic ducts.

Step 3: Decrease of the CFTR expression at the plasma membrane results in a low $\mathrm{HCO}_{3}^{-}$concentration in pancreatic juice.

Step 4: Decrease of $\mathrm{HCO}_{3}^{-}$secretion further decreases the $\mathrm{pH}$ and volume of the fluid secreted by pancreatic ducts.

Step 5: Low fluid volume can result in the precipitation of digestive enzymes in pancreatic fluid and protein plugs are formed in pancreatic ducts in chronic pancreatitis.

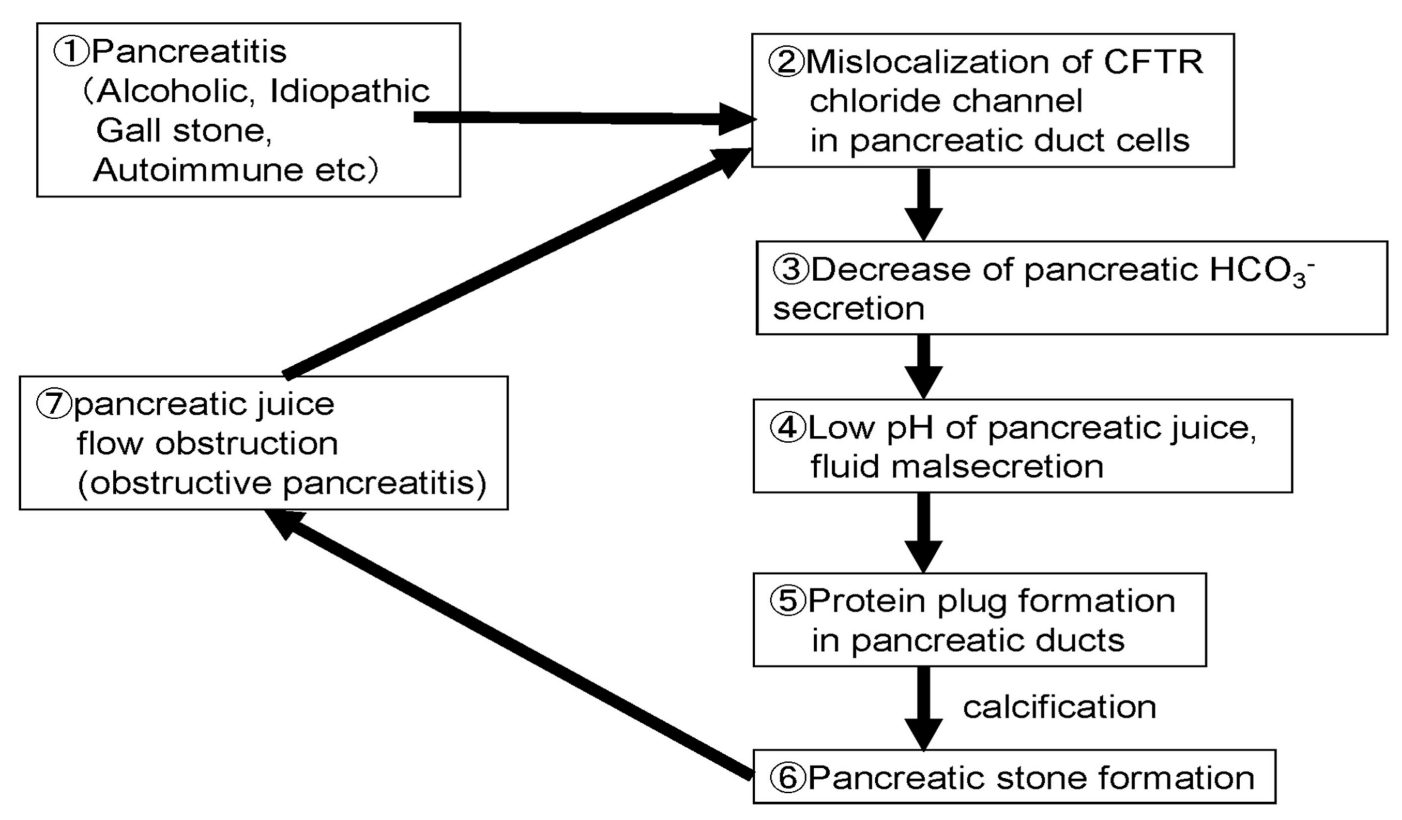

FIGURE 6 | Proposed cellular mechanisms of pancreatic stone formation in chronic pancreatitis. 
Step 6: Protein plugs formed in pancreatic ducts disturb pancreatic juice outflow and protein plugs are calcified.

Step 7: Protein plugs and pancreatic stones obstruct pancreatic juice flow further and cause obstructive/upstream pancreatitis. Obstructive pancreatitis caused by protein plugs and stones in pancreatic ducts exacerbate the cytoplasmic mislocalization of CFTR, and then compromise pancreatic ductal dysfunction.

\section{CONCLUSIONS}

Extensive research has revealed the molecular mechanisms of ion and fluid secretion in the physiological and pathological status

\section{REFERENCES}

Braganza, J., Lee, S., McCloy, R., and McMahon, M. (2011). Chronic pancreatitis. Lancet 377 , 1184-1281.

Burghardt, B., Elkaer, M. L., Kwon, T. H., Racz, G. Z., Varga, G., Steward, M. C., et al. (2003). Distribution of aquaporin water channels AQP1 and AQP5 in the ductal system of the human pancreas. Gut 52, 1008-1016.

Freedman, S. D. (1998). New concepts in understanding the pathophysiology of chronic pancreatitis. Int. J. Pancreatol. 24, 1-8.

Frulloni, L., Scattolini, C., Katsotourchi, A. M., Amodio, A., Gabbrielli, A., Zamboni, G., et al. (2010). Exocrine and endocrine pancreatic function in 21 patients suffering from autoimmune pancreatitis before and after steroid treatment. Pancreatology 10, 129-133.

Furuya, S., Naruse, S., Ko, S. B., Ishiguro, H., Yoshikawa, T., and Hayakawa, T. (2002). Distribution of aquaporin 1 in the rat pancreatic duct system examined with lightand electron-microscopic immunohistochemistry. Cell Tissue Res. 308, 75-86.
Homma, T., Harada, H., and Koizumi, M. (1997). Diagnostic criteria for chronic pancreatitis by the Japan Pancreas Society. Pancreas 15, 14-19.

Ishiguro, H., Steward, M., Naruse, S., Ko, S., Goto, H., Case, R., et al. (2009). CFTR functions as a bicarbonate channel in pancreatic duct cells. J. Gen. Physiol. 133, 315-341.

Ito, T., Kawabe, K., Arita, Y., Hisano, T., Igarashi, H., Funakoshi, A., et al. (2007). Evaluation of pancreatic endocrine and exocrine function in patients with autoimmune pancreatitis. Pancreas 34, 254-259.

Ko, S., Mizuno, N., Yatabe, Y., Yoshikawa, T., Ishiguro, H., Yamamoto, A., et al. (2010). Corticosteroids correct aberrant CFTR localization in the duct and regenerate acinar cells in autoimmune pancreatitis. Gastroenterology 138, 1988-2084.

Ko, S., Shcheynikov, N., Choi, J., Luo, X., Ishibashi, K., Thomas, P., et al. (2002). A molecular mechanism for aberrant CFTR-dependent $\mathrm{HCO}_{3}^{-}$ transport in cystic fibrosis. $E M B O \mathrm{~J}$. 21, 5662-5734.

Ko, S., Yamamoto, A., Azuma, S., Song, H., Kamimura, K., Nakakuki, M., et al. (2011). Effects of CFTR gene

(chronic pancreatitis) of the pancreas. Cytoplasmic mislocalization of the CFTR chloride channel results in aberrant $\mathrm{HCO}_{3}^{-}$secretion from the pancreatic ducts in chronic pancreatitis. Nonetheless, there is no cure for pancreatic ductal dysfunction in $\mathrm{CP}$ such as alcoholic or idiopathic pancreatitis. However, we have found for the first time that pancreatic ductal dysfunction of patients with autoimmune pancreatitis was partially reversed by the corticosteroid treatment. Further investigation to examine the effects of an anti-inflammatory regimen on pancreatic ductal dysfunction in other forms of Cp should eventually lead to the establishment of the treatment for chronic pancreatitis.

silencing by siRNA or the luminal application of a CFTR activator on fluid secretion from guinea-pig pancreatic duct cells. Biochem. Biophys. Res. Commun. 410, 904-913.

Ko, S., Zeng, W., Dorwart, M., Luo, X. Kim, K., Millen, L., et al. (2004). Gating of CFTR by the STAS domain of SLC26 transporters. Nat. Cell Biol. 6 , 343-393.

Mizuno, N., Bhatia, V., Hosoda, W. Sawaki, A., Hoki, N., Hara, K., et al. (2009). Histological diagnosis of autoimmune pancreatitis using EUS-guided trucut biopsy: a comparison study with EUS-FNA. J. Gastroenterol. 44, 742-792.

Song, Y., Yamamoto, A., Steward, M. C. Ko, S. B., Stewart, A. K., Soleimani, M., et al. (2012). Deletion of Slc26a6 alters the stoichiometry of apical $\mathrm{Cl}^{-}$ $\mathrm{HCO}_{3}{ }^{-}$exchange in mouse pancreatic duct. Am. J. Physiol., Cell Physiol. 303, C815-C824.

Steward, M., Ishiguro, H., and Case, R. (2005). Mechanisms of bicarbonate secretion in the pancreatic duct. Annu. Rev. Physiol. 67, 377-786.

Yoshida, K., Toki, F., Takeuchi, T. Watanabe, S., Shiratori, K., and Hayashi, N. (1995). Chronic pancreatitis caused by an autoimmune abnormality. Proposal of the concept of autoimmune pancreatitis. Dig. Dis. Sci. 40, 1561-1568.

Conflict of Interest Statement: The authors declare that the research was conducted in the absence of any commercial or financial relationships that could be construed as a potential conflict of interest.

Received: 05 September 2012; paper pending published: 25 September 2012; accepted: 10 October 2012; published online: 05 November 2012.

Citation: Ko SBH, Azuma S, Yoshikawa T, Yamamoto A, Kyokane K, Ko MSH and Ishiguro H (2012) Molecular mechanisms of pancreatic stone formation in chronic pancreatitis. Front. Physio. 3:415. doi: 10.3389/fphys.2012.00415

This article was submitted to Frontiers in Gastrointestinal Sciences, a specialty of Frontiers in Physiology.

Copyright (c) 2012 Ko, Azuma, Yoshikawa, Yamamoto, Kyokane, Ko and Ishiguro. This is an open-access article distributed under the terms of the Creative Commons Attribution License, which permits use, distribution and reproduction in other forums, provided the original authors and source are credited and subject to any copyright notices concerning any third-party graphics etc. 